\section{Cahiers de Narratologie}

Analyse et théorie narratives

$11 \mid 2004$

Figures de la lecture et du lecteur

\title{
Catulle et ses lecteurs
}

\section{Évrard Delbey}

\section{OpenEdition}

Journals

Édition électronique

URL : http://journals.openedition.org/narratologie/8

DOI : 10.4000/narratologie.8

ISSN : 1765-307X

Éditeur

LIRCES

\section{Référence électronique}

Évrard Delbey, «Catulle et ses lecteurs », Cahiers de Narratologie [En ligne], 11 | 2004, mis en ligne le 01 janvier 2004, consulté le 10 décembre 2020. URL : http://journals.openedition.org/narratologie/8 ; DOI : https://doi.org/10.4000/narratologie.8

Ce document a été généré automatiquement le 10 décembre 2020.

\section{(c) (i) (9)}

Cahiers de Narratologie - Analyse et théorie narratives est mis à disposition selon les termes de la licence Creative Commons Attribution - Pas d'Utilisation Commerciale - Pas de Modification 4.0 International. 


\title{
Catulle et ses lecteurs
}

\author{
Évrard Delbey
}

A la mémoire de J. Granarolo, ce Catullien vivant.

1 Nous inscrivons cette étude dans un sujet plus vaste: la construction de plusieurs types de lecteurs par un auteur. Nous avons choisi la figure d'un poète latin du 1er siècle av. J.C. pour analyser cette diversité qui agit sur les processus de réception d'un recueil de Poèmes et dont l'un des aspects consiste en la figure du poète lecteur de lui-même, devenu autre par l'énonciation de son nom propre. Nous organiserons ce travail de manière à mettre en perspective ce sentiment d'altération de l'identité morale. La traduction choisie est celle d'André MARKowicz, Le livre de Catulle, L'Age d'Homme, 1985; le texte reste celui de la Collection des Universités de France.

2 Ce ne sont pas les questions portant sur la réception de l'oeuvre de Catulle qui nous préoccupent dans ce travail; autrement dit, nous n'étudierons pas les jugements formulés par Properce ou Ovide -pour ne prendre que l'exemple de poètes partageant une thématique élégiaque- sur la poésie catullienne. Nous nous intéresserons plutôt aux diverses présences de lecteurs dans les Poèmes selon que prédominent des relations d'amitié, d'inimitié, de passion amoureuse; de fait, le plus souvent Catulle adresse directement son poème à un destinataire précis avec lequel il entretient un rapport individuel.

3 Toutefois, nous mettons à part le premier poème du recueil : dédicace de l'ouvrage à Cornelius Nepos, ami du poète, historiographe s'intéressant aux hommes illustres tels Agésilas, Alcibiade, Aristide, Atticus, Caton, Cimon, Dion de Syracuse, Epaminondas, Hamilcar, Hannibal, Lysandre, Miltiade, Pausanias, Pélopidas, Phocion, Thémistocle, Thrasybule... Poète à ses heures, il édita peut-être le manuscrit de Catulle. Le ton des vers est affectueux et place Cornelius dans la position du lector doctus qui sait reconnaitre les mérites de quelques petits vers :

"A qui donc le donner, ce petit livre

Tout nouveau, policé de pierre ponce? -

A toi seul, Cornélius, qui semblais prendre

Mes petits musements pour quelque chose

(...) 
Daigne donc l'accepter, ce tendre livre,

Quoi qu'il vaille ... (...)." (c.1, 1-4, 8-9).

4 Cet envoi suppose un acte de lecture idéale où seul Cornelius Nepos jouit de la possibilité de lire tous les poèmes, alors que le recueil présente une multitude de lecteurs nommés et isolés par les vers qui les désignent. Catulle recourt à l'autorité d'une dédicace qui officialise, en le simplifiant, le rapport au texte poétique; au-dessus des lecteurs que l'existence sépare, la personne d'un lecteur archétypal, chargé d'accueillir la plurivocité, l'hétérogénéité des textes; lecteur sinon unique, du moins premier.

5 Nous commençons, donc, par les références aux lecteurs-amis, dès lors que le recueil existe grâce à cette proximité due à l'intimité partagée de la vie privée. Ainsi le c.6 somme gentiment Flavius d'accepter de confier à Catulle le détail de ses ébats amoureux :

"Mon Flavius, il faudrait que tu me parles,

Tu ne pourrais te taire si ta belle

Ne manquait ni de charme ni de grâce,

Mais tu aimes je ne sais quelle pute

Épuisée, -tu as honte de le dire.

(...)

Ainsi donc, que tu sois ou non à plaindre,

Parle, -et toi comme celle que tu aimes,

Que le charme du chant vous porte au ciel."

(c.6, 1-5, 15-17).

6 Nous trouvons un motif analogue dans le c.55 à Camerius. Nous voyons immédiatement que la poésie de Catulle n'est pas écrite pour elle-même; elle suppose l'existence d'un lecteur qu'elle nomme et qu'elle interpelle; nous évoluons dans un espace littéraire qui reproduit la communication orale: cela fut analysé en son temps par J. GranARoLo. Destinataire le plus souvent unique, le lecteur n'est pas interchangeable. Autre exemple, le c.9 qui dit l'exultation saisissant le poète au moment de revoir son cher Veranius :

"Véranius, le meilleur des camarades,

Le plus proche entre trois cent mille proches,

Tu reviens t'incliner à tes pénates,

Chez tes frères chéris, ta vieille mère,

Tu reviens, sain et sauf ... C'est une fête!

Tu viendras me charmer par l'Hibérie,

Ses nations, leur histoire, leurs coutumes

Et je t'embrasserai, enfin, sans trêve,

Sur le cou, sur les yeux et sur les lèvres ...

Qui des hommes heureux pourrait se dire

Plus heureux et plus gai que ton Catulle?"

7 Cette tendresse marque également l'invitation au festin envoyée à Fabullus qui, par son inspiration, reprend la poésie de banquet dont la tradition est grecque; nous pénétrons, alors, dans un petit monde de plaisirs partagés; lecteur hédoniste de ce c.13, Fabullus ne nous sera connu que par son goût de la vie joyeuse :

"Tu feras chez Catulle, Fabullus,

Un festin, si les dieux sont favorables

Et si tu nous amènes de quoi faire

Ce festin, -une fille éblouissante,

De l'esprit et du vin et tous les Rires.

Si tu prends tout cela, gracieux jeune homme,

$\mathrm{Tu}$ feras un festin puisque les toiles

D'araignée règnent seules dans ma bourse.

En échange, reçois le pur Amour 
Et le plus élégant, le plus aimable

Des cadeaux : un parfum que mon amante

A reçu de Vénus et Cupidon.

Tu prieras que les dieux, mon Fabullus,

Te transforment en nez à ces essences."

8 Ainsi composé, ce poème épigrammatique est un billet qui semble attendre sa réponse.

Calvus, de son côté, orateur et poète célèbre, pleura dans un recueil d'élégies son épouse Quintilia; Catulle écrit pour lui le c.96 où s'expriment tout le tact et la beauté d'une compassion qui se veut porteuse de consolation:

"S'il se pouvait que les tombes, pourtant muettes, éprouvent

Quelque agrément à sentir notre tristesse, Calvus,

Dans le tourment des regrets qui revit nos amours de jeunesse

Quand nous pleurons l'amitié morte pour nous dès longtemps,

Quintilia serait moins affligée d'être morte si jeune

Que bienheureuse en voyant comme tu l'aimes toujours."

9 Cette courte élégie montre elle aussi que le poète n'envisage de public que composé de ceux dont il se sent le plus proche, tant que ses relations sont placées sous le signe de la confiance amicale et du dévouement. Cela permet des variations de ton dans ce registre positif : sourire, joie, amusement, tristesse évitant de peser trop, autant d'instantanés de l'écriture et de la vie. Le c.50 complète l'image de la force de l'amitié pour Licinius Calvus. Le billet érotique à Ipsithilla marque, à sa manière, cette évidence de l'existence qui devient la texture de la parole littéraire; c'est le c.32 :

"J'aimerais, toute douce Ipsithilla,

Mes charmantes jouissances, mes délices,

Qu'à midi tu m'invites dans ta chambre.

Et si tu m'y invites, sois gentille,

Ne mets pas le loquet contre la porte,

N'essaie pas de sortir avant que j'entre

Et attends, bien au calme, dans ta chambre,

Prête à être foutue neuf fois de suite.

Mais alors, que ce soit à l'instant même,

Car, repus, reposé, la panse pleine,

Je transperce déjà tunique et cape."

10 L'écriture coïncide avec ce moment d'impatience amoureuse. Nous terminerons cette première série d'exemples par le c.56. P.Valerius Caton, auteur de poèmes mythologiques et érotiques voire obscènes, y occupe la place de lecteur privilégié car instruit avant tout le monde d'une scène osée :

"Ah, Caton, cette blague était si drôle

Et si digne pour toi d'éclats de rire!

Ris, Caton, toi qui aimes ton Catulle;

Cette blague, vraiment, était trop drôle.

J'ai surpris un garçon trouant sa garce

A grands coups, et, pour plaire à la déesse,

J'ai percé, tout rigide, ses arrières."

11 Ni la chasteté ni la vertu ne sont de mise ici; la brutalité tient au mode d'énonciation tout autant qu'à la désignation même du lecteur qui se veut provocante; le caractère public que prend la transgression audacieuse de la morale collective se fonde, en effet, sur une ambiguité : Catulle joue sans doute sur l'homonymie qui fait penser au respectable Caton d'Utique, lorsque P.Valerius Caton est interpellé. Ainsi, le poème trouve une part de sa force dans ce quiproquo possible à propos de son destinataire. 

les figures de lecteurs nommés permettent de concilier les références au réel avec la poésie; écrits de manière "réaliste", les carmina n'excluent pas l'"autoréflexivité", Catulle concevant sa poésie comme la mise en scène d'un lecteur-destinataire qui le renvoie à luimême, à ses sentiments. À ce titre, nous pouvons évoquer le lecteur double de l'auteur et le poème miroir tendu où l'ami se reconnaît.

Il n'en va pas de même lorsque Catulle s'en prend à ses ennemis : les lecteurs-ennemis sont l'objet de toutes les invectives. Aurelius et Furius l'apprennent plusieurs fois à leurs dépens :

"Je vous baise, putain, je vous transperce, Aurélius le pédé, Furius la pute!

Pour des vers délicats, trop peu pudiques,

M'accuser de sombrer dans la débauche?!..

En son corps, le poète pieux est chaste

Mais dans ses petits vers c'est inutile

Car ils n'ont du piquant et de la grâce

Que s'ils sont délicats et peu pudiques,

S'ils éveillent encore les désirs

-Pas des gosses- des hommes bien poilus

Qui ne peuvent bouger leurs reins trop lourds.

Me traiter de fillette si je parle

De milliers de baisers dans mes poèmes?!...

Je vous baise, putain, je vous transperce!"

c.16, qui est à lire avec les c.15, 19, 23, 26 contre les mêmes, est paradoxal : tout en affirmant la distinction entre le Je du poète et le Moi de l'individu, il représente Aurelius et Furius en ennemis personnels, cibles des vers. Constat identique à propos du virulent c.29 qui attaque Mamurra, aide de camp et ami de César, commandant du génie pendant la guerre contre les Gaulois : il s'était enrichi à force de rapines et Pline raconte qu'il fut le premier Romain à n'avoir que des colonnes en marbre dans son palais; Catulle ne cesse de le surnommer Verge (cf. les c.115, 114, 105, 94, 57) et n'hésite pas à insulter César et Pompée, pour quelques temps encore alliés politiques; ces lecteurs illustres, mentionnés par le c.29, signalent l'enracinement historique, voire idéologique, d'une poésie si proche alors des "graffiti" :

"Qui peut le voir, cela, qui peut le supporter,

A moins d'être un joueur glouton et perverti,

Que Mamurra possède ce que possédaient

La Gaule Chevelue et toutes les Bretagnes?

Roulure-Romulus, peux-tu le voir, cela?" (v.1-5).

Le poème continue dans le même débordement de mots insolents. Les c.54 et 93, plus spécifiquement dirigés contre les césariens et leur chef, transforment l'espace littéraire en terrain d'affrontement public. Et Cicéron lui-même n'échappe pas aux emportements d'un poète devenu satirique. C'est le fameux c.49:

"Noble, oratorissime fils de Rome,

Chez les morts, les vivants, Marcus Tullius,

Et les rhétoriqueurs encore à naître,

Ton Catulle te rend des tas de grâces,

Lui, le plus méprisable des poètes ...-

Tout aussi méprisable, ce poète,

Que tu es l'avocat le plus louable." 
L'ironie n'est pas déguisée : le compliment final est excessif, réparant mal l'emphase volontairement ridicule du premier vers. De fait, Cicéron n'appréciait pas les nouveaux poètes parmi lesquels Catulle et Calvus étaient les plus célèbres. Au-delà de l'animosité des personnes, une querelle d'esthétique: l'orateur ne peut lire les Poèmes_avec bienveillance, il n'y trouve pas la beauté de la grandeur morale qui suscite le sublime.

Nous sommes, donc, dans une poésie où l'objet de la représentation est la situation récurrente de la relation auteur/lecteur; la classique hiérarchisation qui, héritage de la Poétique d'Aristote, fait de la tragédie et de l'épopée les deux genres majeurs, par opposition à ces genres mineurs que sont la comédie, l'élégie, la poésie lyrique et la satire, est fortement mise à distance : un poète peut être doctus tout en privilégiant dans la vie réelle l'expérience individuelle au quotidien et non les grands moments intéressant la collectivité, qu'ils soient légendaires ou socio-historiques. Catulle abaisse même les personnages de rang social élevé qu'il représente, tels César ou Cicéron, en les comptant au nombre de ses propres ennemis. Nous assistons à une dévalorisation volontaire de la poétique aristotélicienne, si nous entendons par ce terme un art de l'évocation imaginaire qui prend pour modèles les sujets et le style épico-tragique, et de la rhétorique cicéronienne, si nous désignons de la sorte un art de la communication politique selon les principes philosophiques du beau et du bon. Même si Catulle adopte le ton qui convient selon la personne à laquelle il destine son poème, il ne respecte pas la convenance esthétique qui vise l'universel par la représentation héroïque de l'humain. Cependant, le poète sait mettre en forme des effets de contraste, il joue la variété des sentiments contre la convenance. L'omniprésence des lecteurs de Catulle n'est pas innocente, mais indique une évolution des goûts littéraires; nous l'interprétons comme une marque de l'émergence de l'individuel dans le poétique.

Le c.30 insiste sur cette nouveauté. Alfenus n'a pas en amitié la fidélité d'un Veranius ou d'un Calvus; le chagrin qu'en éprouve Catulle prouve amèrement que la poésie ne peut plus être harmonieuse; à chaque état d'âme ou presque correspond un ton.

Nous abordons maintenant des textes où l'amitié persiste sans pourtant exclure la souffrance et la plainte; par eux, nous saisirons mieux encore ce que signifie une esthétique du contrepoint et de la lecture plurielle, puisque, dans un même poème et non plus d'un poème à l'autre, les émotions se mêlent. La figure du lecteur s'enrichit de cette capacité de s'adapter à la diversité. Le c.65 inaugure la part des Poèmes écrite en métrique élégiaque; il annonce le c.66, inspiré de Callimaque, sur la Boucle de Bérénice. Adressée à Ortalus, cette élégie dit à la fois la douleur, l'absence de la douce inspiration des Muses et le devoir de tendre amitié qui pousse Catulle à envoyer quand même le c.66 promis à Ortalus. Longuement traversé par la peine accablante que continue de produire la mort $d u$ frère du poète, le texte est mythologique par la référence à Philomèle déplorant l'assassinat de son fils, personnel par la pensée du frère disparu aux pieds tout pâles que baigne le Léthé, érotique par la gracieuse mise en scène d'une jeune fille avouant malgré elle à sa mère qu'elle a un fiancé déclaré. Ces trois registres sont trois images complémentaires du style élégiaque qui, ainsi, suppose trois modes de lecture: celui, originel, du chant de deuil; celui, romain, du pathétique subjectif; celui, alexandrin, de la passion amoureuse. Une harmonie de contrastes se crée alors et le style élégiaque réunit diverses manières d'écrire, donc de lire le même sujet.

Le c.68 à Manlius reprend ce principe. Apparemment trompé dans ses espoirs de bonheur en amour, Manlius attend de Catulle le réconfort; l'élégie répond à un "billet épuisé", tout 
humecté de pleurs; mais le poète est toujours aussi abattu par le malheur dû à la perte de son frère; alors la consolation de l'autre paraît impossible à celui qui se sait lui-même inconsolable et Catulle, retiré à Vérone, loin de Rome et de ses amours, n'a rien à envoyer pour soulager l'affliction de son ami (v.1-40). Le mode de lecture dominant est le "pathos". Cependant, la longue suite du poème (v.41-162) introduit l'érôs par le rappel de l'amitié dont Manlius fit preuve en favorisant les amours de Catulle; l'éloge de l'ami et de l'amour se confondent: le poète retrouve le ton de l'élégie alexandrine pour décrire l'arrivée de l'aimée (v.133-134 après les vers 67-72). Mais, en songeant à la passion légendaire de Laodamie pour Protésilas, Catulle se souvient de l'enlèvement d'Hélène et de la funeste guerre de Troie, provoquant le retour lancinant de l'image de son frère mort en terre troyenne précisément. Subtilement, l'élégie progresse dans cet entrelacement de bonheurs et de malheurs; l'érôs finit par triompher, le texte se conclut sur l'éloge de l'adultère (v.161-162). Catulle donne donc une élégie qui célèbre la vie par une série de topiques croisées.

Aussi devons-nous étudier à présent les poèmes à l'aimée, Lesbie douce et cruelle; ils sont animés de cette complexité de sentiments où le lyrisme intense entre en conflit avec le scatologique. Il suffit de mettre bout à bout quelques poèmes pour que cette belligérance du texte apparaisse.

"Vivons donc, ma Lesbie, aimons, te dis-je,-

Que les voix des vieillards les plus austères

Nous dérangent autant que vent qui passe.

Le soleil peut mourir, s'il doit renaître,-

Nous, que meure, une fois, notre étincelle,

Nous avons à dormir la nuit des siècles.

Donne-moi cent baisers, et mille ensuite,

Et un autre millier, et puis cent autres,

Et encore un millier, et cent encore,

Pour qu'à force d'accumuler les mille

Pour nous-mêmes s'embrouille leur décompte

Et qu'un homme méchant ne nous jalouse

A connaître le nombre des baisers" (c.5);

"Tu me disais autrefois ne connaitre qu'un homme -Catulle-

Et le laisser sans rival, fût-ce, Lesbie, Jupiter.

Je t'ai aimée alors non pas comme on aime une amante,

Mais comme un noble père aime son gendre et son fils.

Je te connais aujourd'hui, et bien que le feu me dévore,

Tu me parais tout à coup sans importance, sans prix.

Est-ce possible? dis-tu. L'amant, devant tant de bassesse,

Aime toujours plus fort, mais ne peut plus respecter" (c.72);

"J'aime et je hais. Tu demandes peut-être comment c'est possible?

Qui peut savoir? C'est ainsi. Moi, je le sens sur ma croix" (c.85);

"Si, soudain, les désirs et les rêves d'un homme s'exaucent,

C'est, contre toute espérance, un véritable bonheur.

C'est ce bonheur, plus précieux que l'or, que j'éprouve moi-même

Quand je te vois, Lesbie, -rêve exaucé- revenir,

Oui, revenir de toi-même, venir contre toute espérance à

Moi. Que la pierre la plus blanche éternise ce jour!

Qui a jamais vécu plus heureux que moi, qui peut dire

Plus que moi que sa vie est comme un rêve exaucé?" (c.107);

"C'est un amour bienheureux, ma vie, que tu me proposes

Pour qu'il dure entre nous jusqu'au-delà de la mort.

Faites, dieux tout-puissants, qu'elle puisse vraiment le promettre,

Que ses paroles lui soient toutes dictées par le coeur 
Et qu'il nous soit permis de mener au terme suprême

Cette éternelle union, lien d'une sainte amitié" (c.109). brisés puis renaissant. Seul le c. 37 dirigé contre Egnatius avilit la maitresse infidèle qu'il montre en prostituée des bas-fonds de Rome; le lieu est une "taverne à putes", le poème serait à graver sur la porte :

"Car cette belle qui a fui mon étreinte,

Aimée comme nulle autre amante en les siècles,

Pour qui j'ai tant livré d'ardentes batailles,

S'asseoit ici. Et vous l'aimez, tous ensemble,

Indignement refaits tout braves, tout riches,

Roulures minuscules des rues étroites,

Et toi surtout, le bon premier des hirsutes,

Enfant de la Celtibérie lapinière,

Toi, Egnatius, fameux par ta barbe opaque

Et par tes dents frottées d'urine hibérique" (c.37, 11-20).

Image composite, Lesbie, lectrice principale, est aussi la uarietas incarnée du monde : sujet d'élégies amoureuses, elle devient l'objet d'épigrammes; image de l'alternance de beauté et de laideur qui fait la fortune de Catulle, "mimèsis" mi-féroce, mi-tendre. L'ami aimé, Juventius, partage avec Lesbie ce statut éthique qui fonde une esthétique de la contradiction (cf. les c.24 et 48 / les c.81 et 99).

Reste Catulle lecteur de Catulle. Lorsque le poète écrit pour lui-même, les insertions de son nom s'effectuent selon deux modes, positif ou négatif. La thématique de cette lecture de soi est la passion pour Lesbie dans ses effets ravageurs. Le c.46 est le seul à exprimer la joie du retour en Italie et la hâte de quitter la province de Bithynie. Nous choisirons comme premier exemple de ce souci de soi inquiet le fameux c.51: imitant le poème de Sappho, Catulle se représente comme poeta doctus et comme individu; il apostrophe, en effet, dans l'écrivain lecteur de la poétesse l'homme privé lisant ce que la pratique de l'émulation littéraire a produit afin de se mettre en garde contre lui-même :

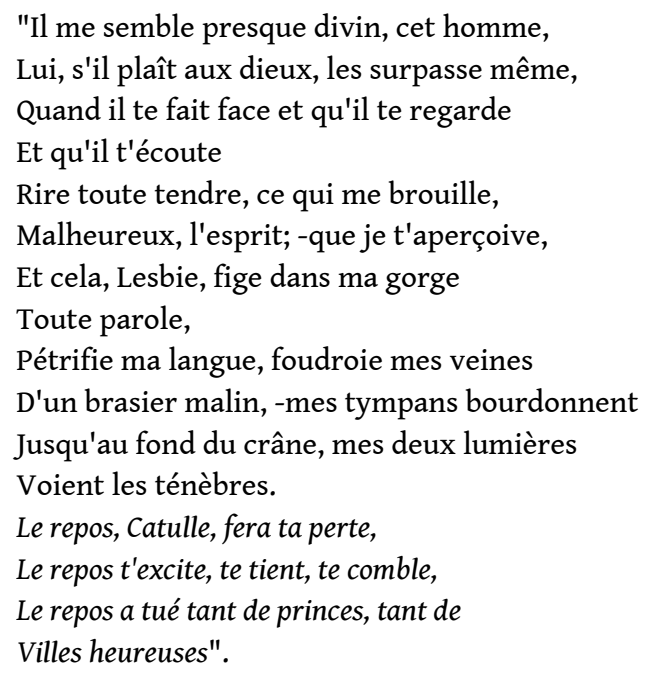

D'abord le poète regarde un homme écoutant le rire charmeur de Lesbie, puis il se décrit regardant Lesbie jusqu'à être aveuglé d'amour par elle, enfin Catulle individu regarde les vers du Catulle poète et prédit sa perte. Ce déplacement progressif de la perspective de lecture modifie les points de vue: le sentiment amoureux, sublime au début et dans le corps du texte, se change en pressentiment pesant de mort. Objet de sa propre représentation, Catulle crée une rupture dans son identité de sujet: il n'est plus 
seulement aveuglé par la passion, mais il est aveuglé/lucide. L'intensité obscure du choc amoureux prend tout son sens par le contraste des derniers vers où le poète voit brutalement clair sur sa condition pitoyable et terrible à la fois. Recul, distance, mise en lumière d'une cécité morale. Catulle dévoile, par la référence intertextuelle à Sappho, l'altération de sa personnalité. Le c.79 évoquera, de fait, le "triste Catulle" (v.2) au moment où le poète reproche à Lesbie d'aimer son propre frère Clodius; l'évidence pathétique que la mémoire intertextuelle de Catulle avait mise en forme dans le c.51, est désormais inaltérable. Le point crucial enfin se trouve atteint, lorsque le poème ne met plus seulement Catulle en présence de lui-même, mais en présence des deux moitiés de sa personnalité partagée entre l'amour et le renoncement à l'amour. Catulle pourfendu. La poétique de la variété n'est pas dissociable du sentiment amer que le poète éprouve de sa dualité intime; le retrait, le repli sur soi constituent une modalité autre de l'énonciation; l'introspection prend la forme de la discordia. Ainsi, de la uarietas à la discordia les Poèmes n'entrent pas dans l'espace défini par Ph.HAMON pour qui tout texte littéraire écrit fonde "une communication différée", donc impossible à réajuster et susceptible de s'adresser à un public hétérogène qui n'est pas "totalement prévisible"("Texte littéraire et métalangage", Poétique n³1, 1977, p.264).

Le c.8 s'organise donc selon une suite d'admonestations que le poète dirige contre luimême. Le premier vers: "Catulle, malheureux, mets fin aux folies" indique la prise de conscience d'un état amoureux désormais révolu; le v.9: "arrête aussi, âme faible" invite au sursaut moral et répond au découragement des vers précédents; le v.12: "tu vois, Catulle tient ferme" est pour Lesbie à laquelle il prédit une existence de femme délaissée; le dernier vers: "Mais toi, Catulle, tu supportes" conforte le poète dans sa décision de renoncer à celle qu'il aima. Le poète se nomme et en écrivant, en lisant son nom, il cerne les limites d'un désastre intérieur. "Folies" (v.1), "mort" (v.2), faiblesse, tristesse sont autant de mots négatifs; "arrête" (v.9), "Ne poursuis plus" (v.10), "vis" (v.10), "reprendstoi un peu, supporte, tiens ferme" (v.11), "tiens ferme" (v.19) sont autant d'expressions positives pour assurer une nouvelle unité, une nouvelle cohésion à l'existence individuelle, ce resaisissement de soi par soi-même. Devenir son propre lecteur permet de maîtriser la passion, maladie de l'âme; l'autoreprésentation participe d'une thérapie du sujet.

Le c.76 reprend cet entretien avec soi-même; Catulle commence par extraire de son passé le souvenir de ses bonnes actions, de sa piété préférable à la torture d'un "amour sans écho" (v.5-6). Face à la résistance du discours amoureux (v.13) le ton de l'énergie se manifeste (v.14-16); finalement ce sont les dieux qui doivent sauver le poète et le poème devient prière (v.17-26). Il a fallu cet exercice de l'écriture-lecture de soi pour que Catulle reconnaisse en lui la part divine du salut moral.

Varietas, discordia nous paraissent des notions susceptibles de résumer les caractéristiques principales de la lecture chez Catulle; nous avons vu comment les relations d'amitié et d'inimitié, d'amour et de haine, l'ambivalence des sentiments font des lecteurs désignés par le poète les destinataires de poèmes aux registres d'expression divers et contradictoires; la personne de l'auteur lecteur de lui-même est là pour représenter le plus fortement possible la désunion intime, le désaccord qui met en péril l'identité harmonieuse du sujet. L'écriture poétique de Catulle est un acte d'énonciation qui a pour principe celui de se savoir lu par tel ou tel.et la lecture, ce moment immédiat de l'apostrophe à l'autre et à soi-même devenant autre. Il y a donc des lectures des Poèmes; elles sont indissociables de la fonction pragmatique de l'énonciation subjective et 
renforçent la forme hyperbolique de l'écriture qui consiste justement à se projeter dans l'espace de sa propre réception.

\section{AUTEUR}

\section{ÉVRARD DELBEY}

Université de Nice 